\title{
SIRT5: A Safeguard Against Oxidative Stress-Induced Apoptosis in Cardiomyocytes
}

\author{
Ban Liu ${ }^{\mathrm{a}, \mathrm{d}}$ Wenliang Che ${ }^{\mathrm{a}, \mathrm{d}}$ Changzhu Zheng ${ }^{\mathrm{b}, \mathrm{d}}$ Weijing Liu Jing Wen $^{\mathrm{a}}$ \\ Haitao Fuc Kai Tang ${ }^{\mathrm{a}}$ Jinying Zhang ${ }^{\mathrm{a}}$ Yawei $\mathrm{Xu}^{\mathrm{a}}$ \\ aDepartment of Cardiology, Shanghai Tenth People's Hospital, Tongji University School of Medicine,

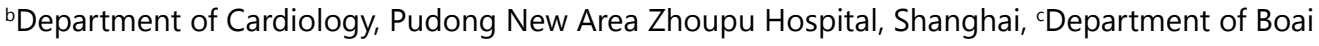 \\ Ophthalmology, Linyi People's Hospital, Linyi, P.R. China; ${ }^{d}$ Contribute equally to this paper as co-first \\ authors
}

\section{Key Words \\ SIRT5 • Mitochondria $\bullet$ Oxidative stress $\bullet B C l-X I$}

\begin{abstract}
Background: SIRT5 is located in the mitochondria, and plays a crucial role in the regulation of metabolic process and cellular apoptosis. Cardiomyocytes are abundant in mitochondria. However, the role of SIRT5 in oxidative stress-induced apoptosis is still unknown in cardiomyocytes. Methods and Results: Western blots analysis revealed that SIRT5 is significantly down-regulated in cardiomyocytes upon oxidative stress. MTT assay, DAPI staining, and caspase 3/7 activity assay were used to estimate apoptosis development. The result suggested that compared with the wild-type group, SIRT5 knockdown results in a marked reduction in cell viability, and a significant increase in the number of apoptotic cells and the caspase 3/7 activity. Protein immunoprecipitation revealed a direct interaction between $\mathrm{Bcl}-\mathrm{XI}$ and SIRT5. Apoptosis assay and western blot anaylsis suggested that SIRT5 levels could affect the levels of $\mathrm{BCl}-\mathrm{Xl}$ expression, but have no effect on the apoptosis development in $\mathrm{BCl}-\mathrm{XI}$ knockdown cells. Conclusion: This study reveals a novel role of SIRT5 in the regulation of oxidative stressinduced apoptosis in cardiomyocytes. Pharmacological interventions on SIRT5 expression may be useful in the treatment of oxidative stress-related cardiac injury.
\end{abstract}

Copyright (C) 2013 S. Karger AG, Basel

\section{Introduction}

Apoptosis is the process of programmed cell death that occurs in multi-cellular organisms. It plays a critical role in regulating cell growth, development, and clearing the redundant cells. Apoptosis could result in cardiomyocyte loss during acute myocardial infarction, 
and subsequent cardiac remodeling events [1]. Cardiomyocytes are terminally differentiated with little potential for division. One strategy to salvage ischemic cardiomyocytes is improving cell survival without disturbing normal cardiac function [2].

Oxidative stress has been implicated in the pathogenesis of many cardiovascular diseases such as ischemic heart disease and heart failure [3, 4]. Excess oxidative stress such as ROS results in DNA damage as well as damage to proteins and lipids, thereby promoting cell death, senescence or aging [5]. Increased ROS is reported to be associated with the dysfunction of myocardial contractility and cardiomyocyte death. It is also a major contributing factor to apoptotic cell death during cardiac ischemia/reperfusion injury and in congestive heart failure [6].

SIRT proteins (sirtuins) are a highly evolutionarily conserved family of NAD-dependent deacetylases [7]. To date, seven SIRT subtypes (SIRT 1-7) have been identified in the mammalian cells. Of them, three sirtuins, SIRT3, SIRT4 and SIRT5, are found to be located in mitochondria [8]. SIRT3 plays a pro-apoptotic role in both Bcl2-53- and JNK-regulated apoptosis. Cells lacking SIRT3 show decreased stress-induced apoptosis, lending further support for a pro-apoptotic role for SIRT3 $[9,10]$. SIRT5 can regulate the expression of carbamoyl phosphate synthetase (CPS1), which is required for removing ammonia generated by amino acid catabolism. SIRT5 binds and deacetylates CPS1, stimulating its enzymatic activity. Mice lacking SIRT5 have elevated ammonia levels after a prolonged fast. Additionally, mice overexpressing SIRT5 show increased CPS1 activity. SIRT5 coordinates the detoxification of hepatic by-products of amino acid catabolism by activating the urea cycle $[11,12]$. Given the physiological role of SIRT5 in metabolic regulation, we hypothesized that SIRT5 may play a critical role in regulating cell viability in cardiomyocytes due to its high abundance in mitochondria in response to oxidative stress.

Here, we used $\mathrm{H}_{2} \mathrm{O}_{2}$ to create oxidative stress condition to induce cardiomyocyte apoptosis in vitro. We provide the evidences that SIRT5 protects cardiomyocyte from $\mathrm{H}_{2} \mathrm{O}_{2}$-induced apoptosis through up-regulating Bcl-Xl expression, suggesting that the physiological and pharmacological regulation of SIRT5 expression may play a critical role in cardioprotective during ischemia/reperfusion injury.

\section{Materials and Methods}

\section{Cell culture and transfection}

H9c2 cells were maintained in Dulbecco's modified Eagle's medium (DMEM) with 10\% calf serum. For transient transfection, H9c2 cells were transfected in 100-mm plates with by using lipofectamine 2000 (Invitrogen) according to manufacturer's instructions.

\section{Isolation of neonatal rat cardiomyocyte}

All procedures for animals were approved by the Animal Ethics and Experimentation Committee of Tongji University (Shanghai, China) and were performed in accordance with the Guide for the Care and the Use of Laboratory Animals published by the US National Institutes of Health (NIH Publication No. 85-23, revised 1996). One-day-old Sprague-Dawley rats were anaesthetized by inhalation of $2 \%$ isoflurane ( $99.9 \%$ from Vedco, St Joseph, MO, USA). The hearts of the neonatal rats were rapidly excised with sharp scissors and then washed with ice-cold PBS to remove blood and debris. After removing the connective tissue, blood vessels and the atria, the ventricles were rapidly minced and incubated in a PBS solution containing trypsin $(0.2 \%)$, collagenase $(0.15 \%)$ and glucose $(0.03 \%)$ for $20 \mathrm{~min}$. The myocardial cells were then isolated by repeat pipetting of the digested myocardial tissue. The cells in the supernatant were transferred into a tube containing culture medium. The tube was centrifuged at $1,000 \mathrm{~g}$ for $10 \mathrm{~min}$ at room temperature, and the cell pellet was re-suspended in the culture medium. Isolated cells were purified by pre-plating for 40 min to reduce the number of non-myocytes. Bromodeoxyuridine was also added to prevent the growth of fibroblasts. Cardiomyocyte purity was approximately $95 \%$ as assessed by microscopic observation of cell beating. 


\section{Western blot}

Cells were lysed with a lysis buffer [50 mM Tris, $50 \mathrm{mM} \mathrm{KCl}, 20 \mathrm{mM} \mathrm{NaF}, 1 \mathrm{mM} \mathrm{Na} \mathrm{VO}_{4}, 10 \mathrm{mM}$ ethylenediaminetetraacetic acid (EDTA), 1\% NP-40, 10 mM nicotinamide, 1 mM TSA, 1 mM phenylmethanesulphonylfluoride (PMSF), $5 \mu \mathrm{g} \mathrm{mL}^{-1}$ leupeptin, $\mathrm{pH}$ 8.0]. Western blot experiments were done after treatment and sample collection. Cell lysate was fractionated by SDS-10\% polyacrylamide gel electrophoresis and transferred to PVDF membranes (Amersham). After blocking with recommended blocking reagents for 1 $\mathrm{h}$ at room temperature, the membranes were incubated overnight at $4{ }^{\circ} \mathrm{C}$ with different antibodies. The membranes were incubated with 1:2000-1:10000 secondary antibodies conjugated with HRP for $1 \mathrm{~h}$ at room temperature after washing for 10 minutes 3 times. Signals were detected by using the Amersham ECL chemiluminescence system.

\section{Analysis of cell viability}

The 3-(4, 5-dimethylthiazal-2-yl)-2, 5-diphenyl-tetrazolium bromide (MTT) assay was used to estimate cell viability [13]. Briefly, cells were plated at a density of $1 \times 10^{4}$ cells per well in 96 -well plates. After exposure to specific treatment, the cells were incubated with MTT at a final concentration of 0.5 $\mathrm{mg} / \mathrm{ml}$ for $3 \mathrm{~h}$ at $37^{\circ} \mathrm{C}$. After removal of the medium, $100 \mathrm{mM}$ DMSO solutions were added to dissolve the formazan crystals. The absorbance at $570 \mathrm{~nm}$ wavelength was detected using a microplate reader (Synergy 4 Hybrid Multi-Mode; BioTek Instruments).

\section{Detection of apoptosis percentage and caspase 3/7 activity}

Apoptosis was induced by $\mathrm{H}_{2} \mathrm{O}_{2}$ treatment. Cells were stained with DAPI and apoptotic nuclei were counted under a fluorescence microscope (300 cells were counted for each experiment). Caspase 3/7 activity was assayed by Promega kit according to the manufacturer's protocol [14, 15]. Caspase-Glo 3/7 Reagent (Promega) was added to all wells in a 1:1 ratio following manufacturer's instructions. After 10 min on a plate shaker at room temperature, $90 \%$ of the lysate volume was transferred to a 96-well solid-white plate. Cell lysates were analyzed with an Analyst HT microplate reader (Molecular Devices), and data points were blank subtracted. Assays were performed in triplicate and are reported as mean \pm S.E.M..

\section{Statistical analysis}

Each experiment was repeated at least three times. The quantitative analysis of Western images was performed using ImageQuant (GE Healthcare). The Statistical significance was determined by the Student's $t$-test. Differences between groups were considered statistically significant if $P<0.05$.

\section{Result}

SIRT5 expression is down-regulated during oxidative stress-induced apoptosis in H9c2 cells

To determine whether SIRT5 expression is altered in response to oxidative stress, H9c2 cells were exposed to $\mathrm{H}_{2} \mathrm{O}_{2}(10,50$, or $100 \mu \mathrm{M})$ for $12 \mathrm{~h}$. The result shows that the administration of $\mathrm{H}_{2} \mathrm{O}_{2}$ up to $50 \mu \mathrm{M}$ results in a statistically significant decrease in SIRT5 expression. Reductions in SIRT5 expression up to $7.6 \%(P>0.05), 52.33 \%(P<0.05)$, and $58.2 \%(P<0.05)$ were detected in the 10,50 , and $100 \mu \mathrm{M} \mathrm{H}_{2} \mathrm{O}_{2}$ groups, respectively (Fig. $\left.1 \mathrm{~A}\right)$. In addition, $\mathrm{H} 9 \mathrm{c} 2$ cells were administrated with $\mathrm{H}_{2} \mathrm{O}_{2}$ at a dose of $50 \mu \mathrm{M}$ for different times. The result indicates that $\mathrm{H}_{2} \mathrm{O}_{2}$ could not lead to a significant increase in SIRT5 expression for the first $5 \mathrm{~h}$, but decreased SIRT5 expression could be detected after $10 \mathrm{~h}$ or $20 \mathrm{~h} \mathrm{H}_{2} \mathrm{O}_{2}$ treatment (Fig. 1B). Taken together, these results suggest that oxidative stress could lead to a reduction in SIRT5 expression in a dose- and time-dependent manner.

Effect of SIRT5 level on the survival of H9c2 cells and neonatal rat cardiomyocytes upon oxidative stress

To investigate the role of SIRT5 in cellular apoptosis, H9c2 cells were transfected with SIRT5 siRNA to suppress SIRT5 expression. As shown in Fig. 2A, SIRT5 siRNA but not scramble siRNA transfection resulted in a marked decrease in SIRT5 expression level. By contrast, 
A
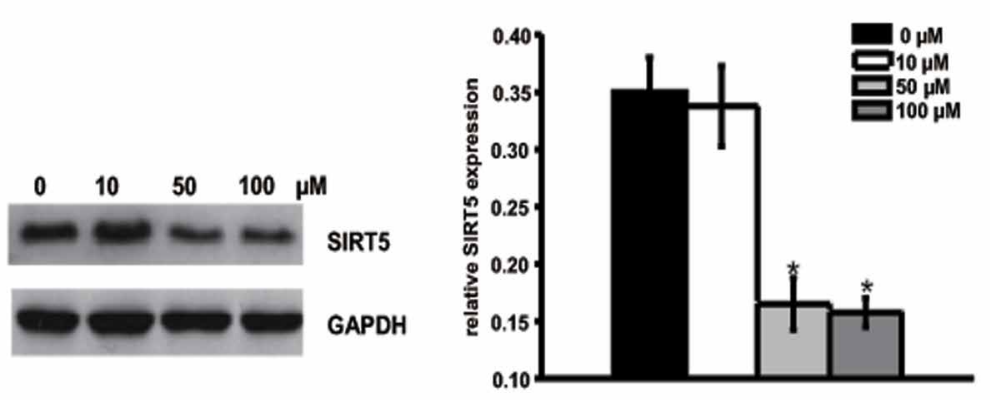

B
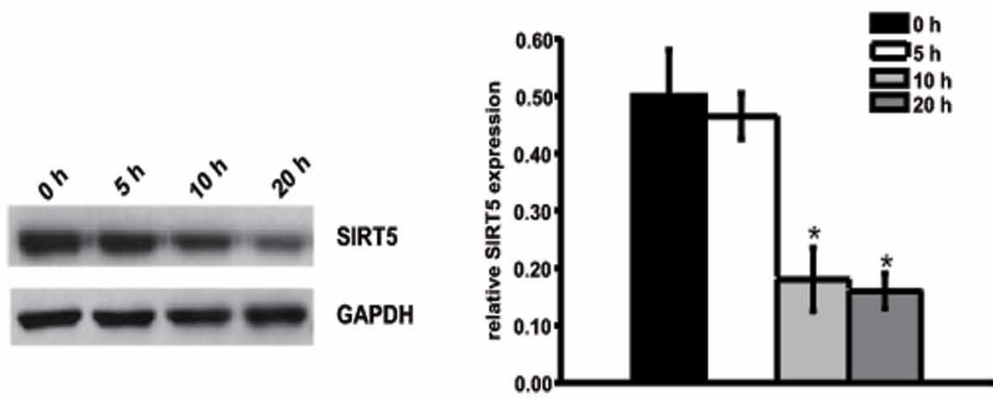

Fig. 1. Aberrant expression of SIRT5 in oxidative stress-induced cardiomyocytes apoptosis. (A) H9c2 cells were treated with $\mathrm{H}_{2} \mathrm{O}_{2}$ at dosed of $0,10,50$, or $100 \mu \mathrm{M}$ for $12 \mathrm{~h}$, and then these cells were lysed. Western blots were conducted to determine the level of SIRT5 expression. GAPDH expression was used as the loading control. (B) $\mathrm{H} 9 \mathrm{c} 2$ cells were treated with $\mathrm{H}_{2} \mathrm{O}_{2}(50 \mu \mathrm{M})$ for different times, and SIRT5 expression was determined as shown in Fig. 1A. SIRT5 expression was shown as the ratio of densitometric value compared to GAPDH expression. Representative immunoblots were shown along with quantitative data showing the mean \pm S.E.M. from four separate blots.

SIRT5 siRNA transfection did not alter the expression of SIRT3 level. Compared with the untreated group, SIRT5 knockdown results in a significant decrease in the cell viability upon oxidative stress (Fig. 2B). To determine whether SIRT5 knockdown results in the reduction in cell viability by promoting cell apoptosis, nuclear staining and caspase- $3 / 7$ activity assay was conducted to detect the change in $\mathrm{H} 9 \mathrm{c} 2$ cell apoptosis upon $\mathrm{H}_{2} \mathrm{O}_{2}$ stress. DAPI staining indicates that the apoptotic cell number significantly increased after $\mathrm{H}_{2} \mathrm{O}_{2}$ treatment, and the effect was further increased by SIRT5 suppression (Fig. 2C). Similarly, $\mathrm{H}_{2} \mathrm{O}_{2}$ treatment dramatically increased caspase-3/7 activity in SIRT5-silencing group, higher than that in control group (Fig. 2D). By contrast, SIRT5 overexpression group has an opposite trend.

To further verify the role of H9c2 cell apoptosis, we employed the pharmacological inhibitor, GW5074, to regulate SIRT5 activity. The result shows that the pharmacological intervention of SIRT5 activity has a similar effect as the molecular intervention of SIRT5 level (Fig. 2B-D). Similarly, the neonatal rat cardiomyocytes were transfected SIRT5 vectors to regulate SIRT5 expression levels. The result also revealed that compared with the wild-type group, SIRT5 knockdown results in a significant decrease in the cell viability, and a marked increase in apoptotic cell numbers and caspase-3/7 activity (Fig.2 E-G). Taken together, these results suggest that SIRT5 plays an important role in the regulation of oxidative stressinduced apoptosis.

SIRT5 overexpression increases Bcl-Xl expression during oxidative stress-induced apoptosis

It is well-known that Bcl-2 family proteins have either pro- or anti-apoptotic activities. They are involved in the execution of apoptosis $[16,17]$. We predicted that SIRT5 may regulate $\mathrm{Bcl}-2$ family expression, which in turn affect the development of cell apoptosis. 
A

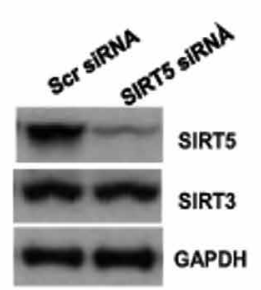

C
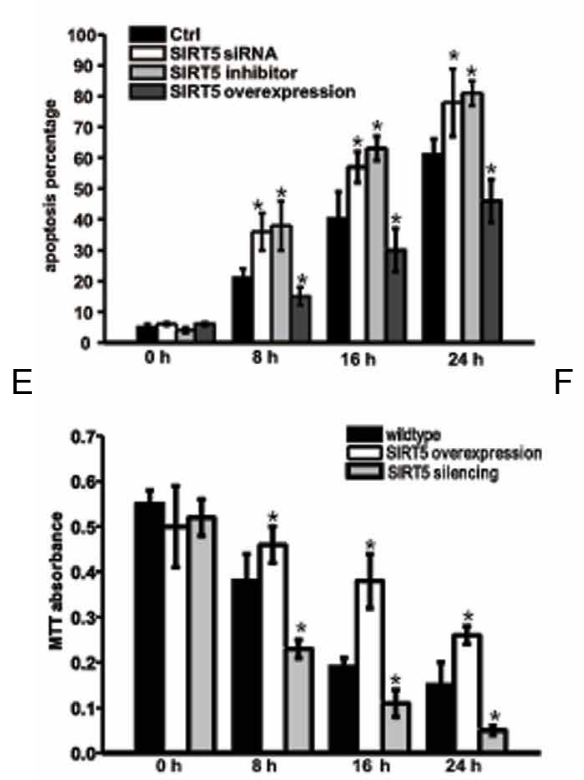

B

$\mathrm{D}$
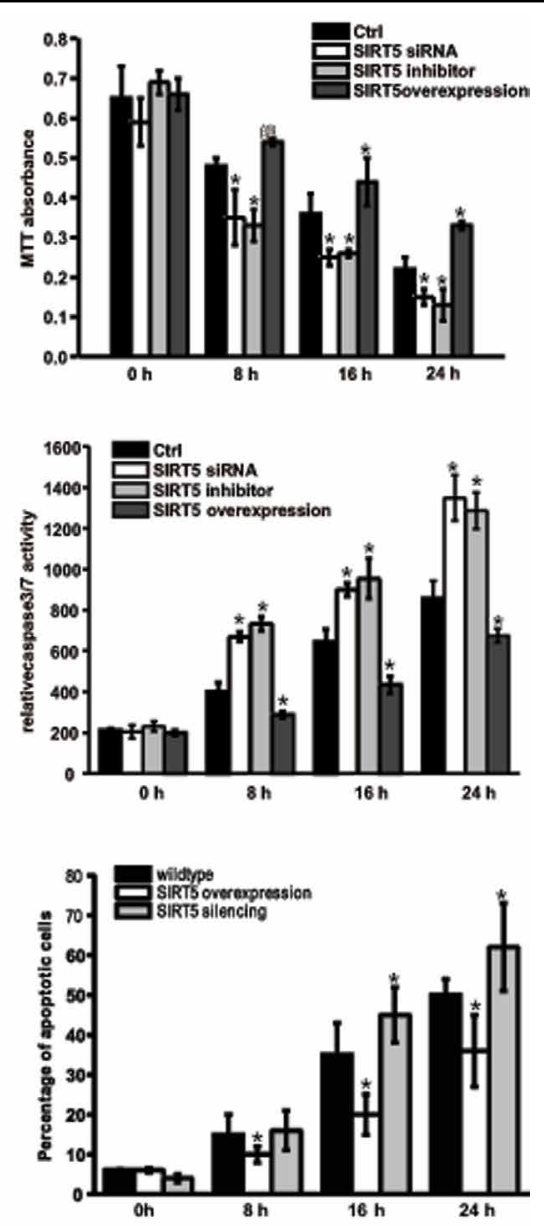

G

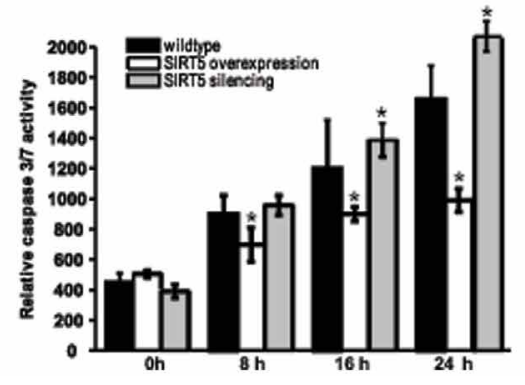

Fig. 2. Effect of SIRT5 on the survival of cardiomyocytes upon oxidative stress. (A) H9c2 cells were transfected with SIRT5 SiRNA, scramble siRNA (Scr) for $24 \mathrm{~h}$. The expression of SIRT5 or SIRT3 was detected by western blot. GAPDH expression was detected as the loading control. Shown is a representative image. (B-G) H9c2 cells or neonatal rat cardiomyocyte apoptosis was induced by $\mathrm{H}_{2} \mathrm{O}_{2}$ at a dose of $50 \mu \mathrm{M}$ for $8 \mathrm{~h}, 16$ $\mathrm{h}$, or $24 \mathrm{~h}$. The untreated cells were used as the control group. The cell viability was determined using MTT assay kit (B and E). The nuclei were stained with DAPI, and apoptotic nuclei were counted under a fluorescence microscope (300 cells were counted for each experiment) (C and F). The activity of caspase 3/7 was determined using caspase 3/7 assay kit (Promega) as described in "Materials and methods" section (D and G). Results are expressed as means \pm S.E.M. of three independent experiments. Each sample was analyzed in triplicate $(P<0.05)$. Asterisk $(*)$ indicates a significant difference compared with the untreated group.

H9c2 cells were first transfected with SIRT5, vector or left untreated, and then they were treated with $\mathrm{H}_{2} \mathrm{O}_{2}$ at a dose of $50 \mu \mathrm{M}$. The untreated group was taken as the control. Western blots analysis demonstrated that $\mathrm{H}_{2} \mathrm{O}_{2}$ treatment resulted in a significant decrease in Bcl-Xl 
Fig. 3. SIRT5 regulates Bcl-Xl expression during oxidative stress-induced apoptosis. H9c2 cell apoptosis was transfected with SIRT5, vector or left untreated for $24 \mathrm{~h}$, and the apoptosis was induced by the treatment with $\mathrm{H}_{2} \mathrm{O}_{2}$ (50 $\mu \mathrm{M}$ ) for $24 \mathrm{~h}$. The untreated cells were used as the control group. Western blots were conducted to determine the levels of Bax, Bcl-2, Bad, and $\mathrm{Bcl}-\mathrm{Xl}$ expressions. GAPDH expression was used as the loading control. Shown is a representative image.

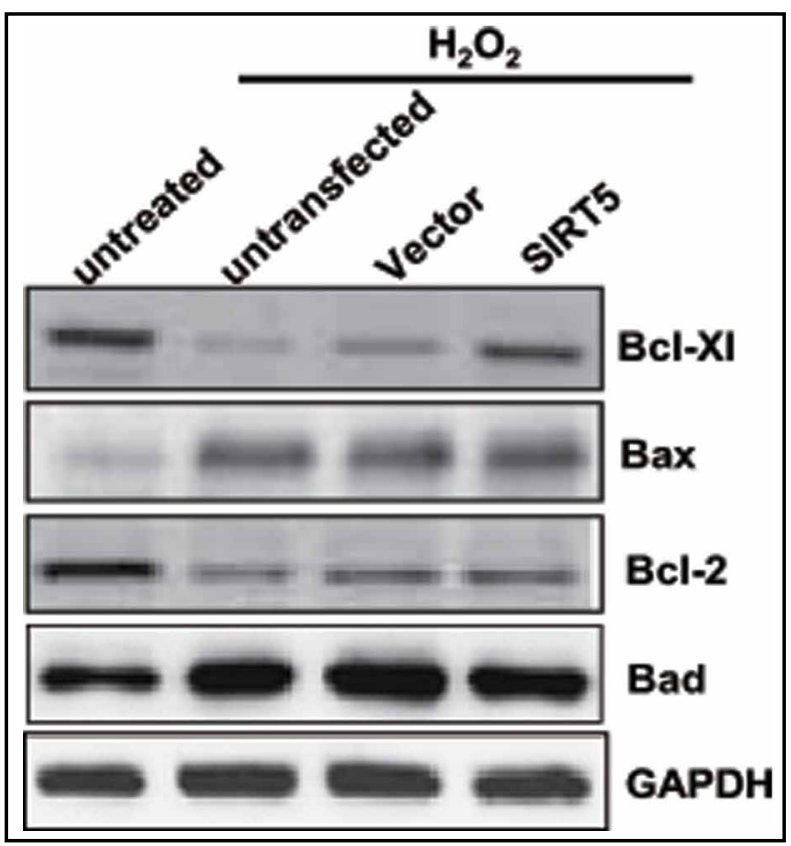

expression, while the reduction in Bcl-Xl expression could be partially reversed by SIRT5 overexpression but not vector overexpression. By contrast, the level of SIRT5 expression had no effect on the expression of Bax, Bcl-2, or Bad upon $\mathrm{H}_{2} \mathrm{O}_{2}$ stress (Fig. 3). Taken together, these evidences suggest that SIRT5 prevents $\mathrm{H}_{2} \mathrm{O}_{2}$-induced cellular apoptosis through the up-regulation of Bcl-Xl expression.

SIRT5/Bcl-Xl interaction is required for the regulation of oxidative stress-induced apoptosis

To corroborate that SIRT5/Bcl-Xl interaction is a critical for $\mathrm{H}_{2} \mathrm{O}_{2}$-induced apoptosis in H9c2 cells, we compared the sensitivity of wild-type and Bcl-Xl deficiency cells to $\mathrm{H}_{2} \mathrm{O}_{2}$ induced cellular apoptosis. The result indicates that $\mathrm{H}_{2} \mathrm{O}_{2}$ treatment results in a significant reduction in cell viability, and a significant increase in apoptotic cell number and caspase-3/7 activity (Fig. 4 A-C). SIRT5 overexpression could partially reverse $\mathrm{H}_{2} \mathrm{O}_{2}$-induced cellular apoptosis in $\mathrm{H} 9 \mathrm{c} 2$ cells. In Bcl-Xl-silencing group, $\mathrm{H}_{2} \mathrm{O}_{2}$ treatment also results in an obvious decrease in cell viability and a significant increase in H9c2 cell apoptosis. However, SIRT5 overexpression could not reverse apoptosis development in Bcl-Xl deficiency cells (Fig. 4 A-C).

To verify whether Bcl-Xl is a direct target of SIRT5, we transected Bcl-Xl and SIRT5 into $\mathrm{H} 9 \mathrm{c} 2$ cells for $24 \mathrm{~h}$. We found that SIRT5 could be immunoprecipitated by Bcl-Xl but not Bak (Fig. 4D). Moreover, we detected an endogenous association between Bcl-Xl and SIRT5 in H9c2 cells (Fig. 4E). Taken together, these results reveal that SIRT5 is a direct target of Bcl-Xl.

\section{Discussion}

Mitochondria play important roles in a variety of biological events, including energy production, calcium buffering and apoptosis regulation. During apoptosis, mitochondria act at the core of the apoptotic pathway by providing many important factors including those that induce caspase activation and chromosome fragmentation. Mitochondria are involved in the production of reactive oxygen species through one-electron carriers in the respiratory chain, particularly in highly metabolically active organs such as the brain and heart $[18,19]$. Thus, these are definitely many proteins existed in mitochondria that are tightly involved in the development of apoptosis. Here, we found that SIRT5, a protein located at the mitochondria, can protect cardiomyocytes from oxidative stress-induced apoptosis in vivo. 


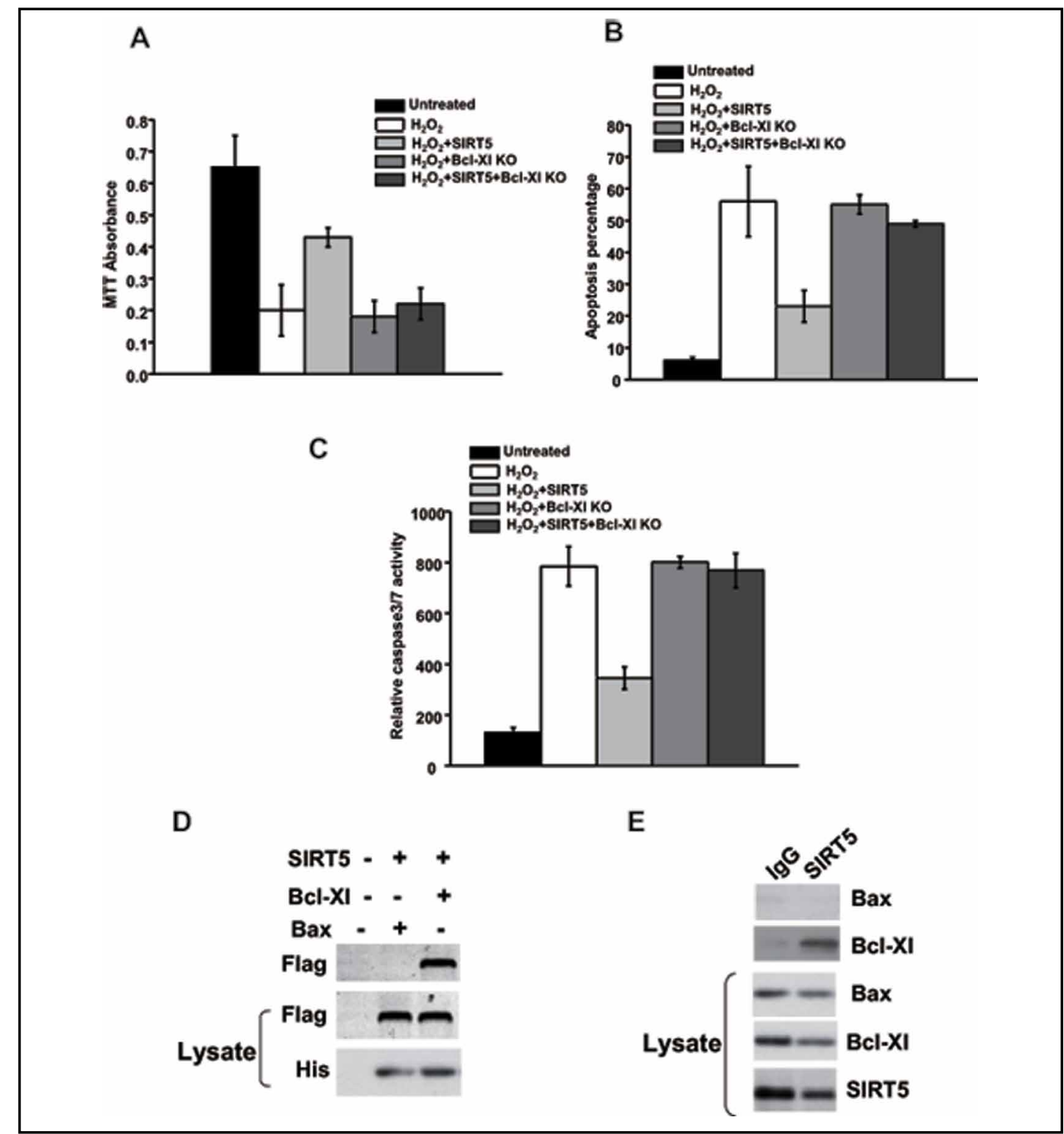

Fig. 4. SIRT5/Bcl-Xl interaction is required for the regulation of oxidative stress-induced apoptosis. (A-C) H9c2 cell was transfected with or without SIRT5 in wild-type cells and Bcl-Xl silencing cells, and then the apoptosis of these cells were induced by the treatment of $\mathrm{H}_{2} \mathrm{O}_{2}$ at a dose of $50 \mu \mathrm{M}$ for $24 \mathrm{~h}$. The untreated cells were taken as the control group. Cell viability was determined using MTT assay kit (A). The nuclei were stained with DAPI, and apoptotic nuclei were counted under a fluorescence microscope (300 cells were counted for each experiment) (B). The activity of caspase $3 / 7$ was determined using caspase $3 / 7$ assay kit (Promega) as described in "Materials and methods" section (C). Results are expressed as means \pm S.E.M. of three independent experiments. Each sample was analyzed in triplicate $(P<0.05)$. Asterisk $\left.{ }^{*}\right)$ indicates a significant difference compared with the untreated group. (D) H9c2 cells were cotransfected with Flag-tagged SIRT5 and His-tagged Bcl-Xl or His-tagged Bax. Forty-eight hours after transfection, these cells were lysed, and then the cell lysates were immunoprecipitated with anti-His antibody. The immunoprecipitates and cell lysates were blotted with the indicated antibodies. (E) The cell lysate of apoptotic H9c2 cells was subjected to immunoprecipitation with anti-IgG or anti-SIRT5 antibody, respectively. The immunoprecipitates were then blotted with the indicated antibodies.

Sir2-like proteins (sirtuins) are a family of NAD-dependent deacetylases conserved from Escherichia coli to humans [20]. They play great roles in a series of biological processes, such 
as gene silencing, DNA repair, rDNA recombination and ageing in model organisms. Of them, SIRT3-5 are found to be located at the mitochondria. SIRT3 plays a pro-apoptotic role in both Bcl2-p53- and JNK-regulated apoptosis. Cells lacking SIRT3 show decreased stress-induced apoptosis, lending further support for a pro-apoptotic role for SIRT3. In conflicting studies, SIRT3 has been shown to be anti-apoptotic. In the cellular response to DNA damage when mitochondrial NAD levels fall below critical levels, SIRT3 and SIRT4 display anti-apoptotic activity, protecting cells from death [21, 22, 23]. Thus, it is crucial to elucidate the balance achieved by sirtuins between stress resistance (anti-apoptosis) and tumor suppression (pro-apoptosis) [24]. Here, we reveal an anti-apoptosis role of SIRT5 in oxidative stressinduced apoptosis in cardiomyocytes. Prior study has suggested that SIRT5 can deacetylate cytochrome $c$, a protein of the mitochondrial intermembrane space with a central function in oxidative metabolism, as well as apoptosis [25], imply a potential role in the regulation of apoptosis. Our study provides the direct evidence that SIRT5 can directly regulate the apoptosis development in cardiomyocytes.

To reveal the role of SIRT5 in cardiomyocytes apoptosis, we used the methods of molecular and pharmacological intervention to regulate SIRT5 activity. We found that any change in SIRT5 expression or its activity is directly associated with the development of $\mathrm{H} 9 \mathrm{c} 2$ cell apoptosis. Sirtuins are $\mathrm{NAD}^{+}$consuming protein deacylases involved in many cellular processes from DNA-repair to metabolism. Their contribution to age-related and metabolic diseases makes them attractive pharmaceutical targets. However, to date, few specific pharmacological inhibitors have been reported yet for human SIRT5. Suenkel et al. (2013) recently reported that the indole GW5074 is a potent inhibitor for SIRT5's desuccinylation activity, identifying a first pharmacological scaffold for development into SIRT5-specific inhibitor [26]. Previous studies also reported that GW5074 could inhibits SIRT2 deacetylation activity with a potency comparable to SIRT (reduction to less than $60 \%$ activity with $12.5 \mu \mathrm{M}$ GW5074) [27, 28]. Although Gw5074 is not a specific inhibitor of SIRT5 deacetylation, its treatment also partially inhibits the SIRT5 deacetylation, providing an indirect evidence that the deacetylation activity of SIRT5 is involved in the apoptosis development of cardiomyocytes.

The anti-apoptotic members such as Bcl-2 and Bcl-Xl are important in cell survival and protect cardiomyocytes against various stressors [29]. For instance, overexpression of Bcl$\mathrm{Xl}$ in $\mathrm{H} 9 \mathrm{c} 2$ cardiac cells protected against doxorubicin- and hypoxia-mediated apoptosis by preserving mitochondrial integrity [30]. Bcl-2 can prevent p53-mediated apoptosis in cardiac myocytes [31]. Transgenic mice overexpressing $\mathrm{Bcl}-2$ in the heart have reduced $\mathrm{I} / \mathrm{R}$ injury and fewer apoptotic cells compared to wild type mice, suggesting that the cardioprotective effect of Bcl-2 is via inhibition of the mitochondrial death pathway $[32,33$, 34]. These studies suggest that the anti-apoptotic proteins can protect against cell death via multiple mechanisms and have attractive therapeutic potential to treat or prevent heart disease. We found that SIRT 5 can directly regulate the level of Bcl-Xl during oxidative stressinduced apoptosis. The role of SIRT5 in regulating apoptosis is found to be disappeared in Bcl-Xl deficiency cell. Thus, we assume that the role of SIRT5 in apoptosis is mainly mediated by the regulation of Bcl-Xl level, and SIRT5 may become an attractive therapeutic target to treat or prevent heart disease.

Mitochondria are the primary ATP provider via the conversion of food fuels to usable energy. The heart is highly rich in mitochondria, and has a continuous requirement for energy in the form of ATP. Compelling evidence for a link between the decline in mitochondrial energy transduction and mechanical dysfunction in heart failure (HF) has been found in animal models and in patients with genetic mitochondrial diseases or acquired cardiomyopathies $[35,36]$. SIRT5 is an important protein located into the mitochondria. It is involved in the regulation of metabolism. Any change in SIRT5 activity may affect the maintenance of energy homeostasis such as energy intake, storage, and expenditure, which in turn results in the occurrence of cardiac diseases $[11,12]$. However, to date, there are still no report about the role of SIRT5 in cardiac diseases. We checked the data for SIRT5 on GEO profiles in regards to cardiac diseases. We found that SIRT5 expression is significantly reduced both 
during isoproterenol-induced cardiomyopathy and exercise-induced cardiac hypertrophy, providing an evidence that SIRT5 is involved in the pathogenesis of cardiac diseases [37]. Thus, SIRT5 may become as a potential target to prevent or treat cardiac diseases.

In summary, this study reveals that SIRT5 is a critical mediator of $\mathrm{H}_{2} \mathrm{O}_{2}$-induced cell apoptosis in cardiomyocytes. SIRT5 knockdown results in a marked reduction in cell viability, and an increase in apoptotic cells number and caspase 3/7 activity. SIRT5 can alter the level of Bcl-Xl expression, thereby changing the development of cardiomyocytes apoptosis. Our study will serve as a start toward deeper understanding of the role of SIRT5 in cardiac diseases. Future studies are required to investigate the correlations between SIRT5 level and the occurrence of cardiac diseases and in vivo functional studies on the effect of SIRT5 on various pathologic pathways of cardiac diseases.

\section{Acknowledgements} to B.L.).

This work was supported by Natural Science Foundation of China (Grant No. 81200131

\section{References}

1 Whelan RS, Kaplinskiy V, Kitsis RN: Cell death in the pathogenesis of heart disease: mechanisms and significance. Annu Rev Physiol 2010;72:19-44.

-2 Van Amerongen MJ, Engel FB: Features of cardiomyocyte proliferation and its potential for cardiac regeneration. J Cell Mol Med 2008;12:2233-2244.

- Minamino T, Kitakaze M: ER stress in cardiovascular disease. J Mol Cell Cardiol 2010;48:1105-1110.

4 Tsutsui H, Kinugawa S, Matsushima S: Mitochondrial oxidative stress and dysfunction in myocardial remodelling. Cardiovasc Res 2009;81:449-456.

5 Roberts CK, Sindhu KK: Oxidative stress and metabolic syndrome. Life Sci 2009;84:705-712.

6 Martindale JL, Holbrook NJ: Cellular response to oxidative stress: signaling for suicide and surviva. J Cell Physiol 2002;192:1-15.

7 Finkel T, Deng CX, Mostoslavsky R: Recent progress in the biology and physiology of sirtuins. Nature 2009;460:587-591.

-8 Verdin E, Hirschey MD, Finley LWS, Haigis MC: Sirtuin regulation of mitochondria: energy production, apoptosis, and signaling. Trends Biochem Sci 2010;35:669-675.

-9 Kim HS, Patel K, Muldoon-Jacobs K, Bisht KS, Aykin-Burns N, Pennington JD, van der Meer R, Nguyen P, Savage J, Owens KM: SIRT3 is a mitochondria-localized tumor suppressor required for maintenance of mitochondrial integrity and metabolism during stress. Cancer Cell 2010;17:41-52.

$>10$ Haigis MC, Deng CX, Finley LWS, Kim HS, Gius D: SIRT3 is a mitochondrial tumor suppressor: a scientific tale that connects aberrant cellular ROS, the warburg effect, and carcinogenesis. Cancer Res 2012;72:24682472.

11 Nakagawa T, Lomb DJ, Haigis MC, Guarente L: SIRT5 Deacetylates carbamoyl phosphate synthetase 1 and regulates the urea cycle. Cell 2009;137:560-570.

-12 Ogura M, Nakamura Y, Tanaka D, Zhuang X, Fujita Y, Obara A, Hamasaki A, Hosokawa M, Inagaki N: Overexpression of SIRT5 confirms its involvement in deacetylation and activation of carbamoyl phosphate synthetase 1. Biochem Bioph Res Co 2010;393:73-78.

13 Gomes LC, Di Benedetto G, Scorrano L: During autophagy mitochondria elongate, are spared from degradation and sustain cell viability. Nat Cell Biol 2011;13:589-598.

14 Deveraux QL, Leo E, Stennicke HR, Welsh K, Salvesen GS, Reed JC: Cleavage of human inhibitor of apoptosis protein XIAP results in fragments with distinct specificities for caspases. EMBO J 1999;18:5242-5251.

15 LeBlanc H, Lawrence D, Varfolomeev E, Totpal K, Morlan J, Schow P, Fong S, Schwall R, Sinicropi D, Ashkenazi A: Tumor-cell resistance to death receptor-induced apoptosis through mutational inactivation of the proapoptotic Bcl-2 homolog Bax. Nat Med 2002;8:274-281. 
16 Valks DM, Kemp TJ, Clerk A: Regulation of Bcl-xL expression by H2O2 in cardiac myocytes. J Biol Chem 2003;278:25542-25547.

17 Gottlieb E, Vander Heiden MG, Thompson CB: Bcl-XL prevents the initial decrease in mitochondrial membrane potential and subsequent reactive oxygen species production during tumor necrosis factor alpha-induced apoptosis. Mol Cell Biol 2000;20:5680-5689.

18 Strassburger M, Bloch W, Sulyok S, Schüller J, Keist AF, Schmidt A, Wenk J, Peters T, Wlaschek M, Krieg T: Heterozygous deficiency of manganese superoxide dismutase results in severe lipid peroxidation and spontaneous apoptosis in murine myocardium in vivo. Free Radical Biol Med 2005;38:1458-1470.

19 Wenzel P, Schuhmacher S, Kienhöfer J, Müller J, Hortmann M, Oelze M, Schulz E, Treiber N, Kawamoto T, Scharffetter-Kochanek K: Manganese superoxide dismutase and aldehyde dehydrogenase deficiency increase mitochondrial oxidative stress and aggravate age-dependent vascular dysfunction. Cardiovasc Res 2008;80:280-289.

20 Imai S, Guarente L: Ten years of NAD-dependent SIR2 family deacetylases: implications for metabolic diseases. Trends Pharmacol Sci 2010;31:212-220.

21 Alhazzazi TY, Kamarajan P, Verdin E, Kapila YL: SIRT3 and cancer: tumor promoter or suppressor? BBA-Rev Cancer 2011;1816:80-88.

22 Zhang Y, Zhou L: Sirt3 inhibits hepatocellular carcinoma cell growth through reducing Mdm2-mediated p53 degradation. Biochem Biophys Res Commun 2012;423:26-31.

23 Pillai VB, Sundaresan NR, Jeevanandam V, Gupta MP: Mitochondrial SIRT3 and heart disease. Cardiovasc Res 2010;88:250-256.

24 Baumann K: Cell death: a pro-apoptotic role for sirtuin 3. Nat Rev Mol Cell Biol 2012;13:750.

25 Schlicker C, Gertz M, Papatheodorou P, Kachholz B, Becker CFW, Steegborn C: Substrates and regulation mechanisms for the human mitochondrial sirtuins Sirt3 and Sirt5. J Mol Biol 2008;382:790-801.

26 Suenkel B, Fischer F, Steegborn C: Inhibition of the human deacylase Sirtuin 5 by the indole GW5074. Bioorg Med Chem Lett 2013;23:143-146.

27 Trapp J, Jochum A, Meier R, Saunders L, Marshall B, Kunick C, Verdin E, Goekjian P, Sippl W, Jung M: Adenosine mimetics as inhibitors of NAD+-dependent histone deacetylases, from kinase to sirtuin inhibition. J Med Chem 2006;49:7307-7316.

28 Lackey K, Cory M, Davis R, Frye SV, Harris PA, Hunter RN, Jung DK, McDonald OB, McNutt RW, Peel MR, Rutkowske RD, Veal JM, Wood ER: The discovery of potent cRaf1 kinase inhibitors. Bioorg Med Chem Lett 2000;10:223-226.

29 Chao DT, Linette GP, Boise LH, White LS, Thompson CB, Korsmeyer SJ: Bcl-XL and Bcl-2 repress a common pathway of cell death. J Exp Med 1995;182:821-828.

-30 Reeve JLV, Szegezdi E, Logue SE, Chonghaile TN, O’Brien T, Ritter T, Samali A: Distinct mechanisms of cardiomyocyte apoptosis induced by doxorubicin and hypoxia converge on mitochondria and are inhibited by Bcl-XL. J Cell Mol Med 2007;11:509-520.

31 Kirshenbaum LA, de Moissac D: The bcl-2 gene product prevents programmed cell death of ventricular myocytes. Circulation 1997;96:1580-1585.

-32 Chen Z, Chua CC, Ho YS, Hamdy RC, Chua BHL: Overexpression of Bcl-2 attenuates apoptosis and protects against myocardial I/R injury in transgenic mice. Am J Physiol-Heart C 2001;280:H2313-H2320.

-33 Lee Y, Gustafsson ÅB: Role of apoptosis in cardiovascular disease. Apoptosis 2009;14:536-548.

-34 Gill C, Mestril R, Samali A: Losing heart: the role of apoptosis in heart disease-a novel therapeutic target? FASEB J 2002;16:135-146.

-35 Neubauer S: The failing heart-an engine out of fuel. N Engl J Med 2007;356:1140-1151.

-36 Doenst T, Nguyen TD, Abel ED: Cardiac metabolism in heart failure: implications beyond ATP production. Circ Res 2013;113:709-724.

-37 Galindo CL, Skinner MA, Errami M, Olson LD, Watson DA, Li J, McCormick JF, McIver LJ, Kumar NM, Pham TQ, Garner HR: Transcriptional profile of isoproterenol-induced cardiomyopathy and comparison to exercise-induced cardiac hypertrophy and human cardiac failure. BMC Physiol 2009;9:23. 